\title{
Early medical abortion - available and safe
}

\section{Early medical abortion using mifepristone should be safely available to all Australian women}

ifepristone, in conjunction with misoprostol, is extremely effective for medical abortion at any gestation and is now widely used throughout much of the world for this purpose. ${ }^{1-3}$ However, the history of the drug in Australia has been controversial. For 10 years from 1996, it was the object of federal legislation that allowed its use in Australia only with the special permission of the federal Minister for Health. Although this legislation was overturned in parliament in February 2006, mifepristone did not immediately become available in Australia - a drug company must receive approval from the Therapeutic Goods Administration (TGA) to import and market it. ${ }^{4}$ To date, this has not happened.

In December 2005, we applied to the TGA under its Authorised Prescriber (AP) regulations to import and use mifepristone for early medical abortion (EMA) in Cairns. These regulations, which are unrelated to abortion, enable individual medical practitioners to import and use drugs that are recognised and used overseas but are unavailable in Australia. Our application was approved and, in mid 2006, we began using mifepristone in Cairns. ${ }^{4}$ We asked colleagues to make similar applications. In 2008, three others received AP approval, and the numbers have since increased to around 200 doctors across Australia.

Extensive overseas studies have shown mifepristone to be both safe and effective. ${ }^{1-3,5,6}$ On this basis, we established protocols using mifepristone $200 \mathrm{mg}$ orally and misoprostol $800 \mu \mathrm{g}$ vaginally $36-48$ hours later. The abortion process, similar to a spontaneous miscarriage, usually takes place within 4-8 hours of misoprostol administration.

As we had no facilities in which the process could occur, this took place in the woman's home. The safety of such arrangements has been well documented in overseas studies; however, it is worth noting that in most countries where EMA using mifepristone-misoprostol has been introduced, this has been initiated in a clinical environment and only later expanded into home abortion services. In the United Kingdom, where mifepristone has been used for 22 years, home abortion is still not widely practised. ${ }^{3,7}$

We require every woman undergoing EMA in Cairns to have a support person, whom we meet and who stays with her throughout the process. We also have in place robust arrangements for emergency care for those who require it, and ensure that women and their support people are familiar with these arrangements. Routine follow-up takes place between 2 days and 2 weeks after abortion and includes the provision of reliable contraception. Evidence to support antibiotic prophylaxis for medical abortion is level C, as no randomised controlled trial has been conducted. However, we have always prescribed prophylactic antibiotics, including cover for chlamydia, as this is our practice for surgical abortion where there is level A evidence supporting it. $^{3}$

Our results, and those published by others, show that mifepristone-misoprostol home abortion is safe, effective and acceptable to Australian women. ${ }^{4,8,9}$ We believe mifepristone can be safely used for EMA by any appropriately trained doctor in Australia, provided there is access to services where spontaneous miscarriage can be safely managed.

However, the numbers of cases reported to date have been relatively small. We therefore welcome the report in this issue of the Journal on the outcomes of more than 13000 EMAs performed in the Marie Stopes International
Caroline M de Costa PhD, FRANZCOG, FRCOG, Professor of Obstetrics and Gynaecology

Michael Carrette MB BS, FRANZCOG Gynaecologist $^{2}$

1 School of Medicine James Cook University, Cairns, QLD.

2 Cairns Private Hospital, Cairns, QLD.

caroline.decosta@ jcu.edu.au

doi: 10.5694/mjal2.10877 
Australia (MSIA) group of clinics over a 2 -year period. ${ }^{10}$ We congratulate MSIA on undertaking the complex administrative processes needed to obtain AP approval for their many doctors. Their success has made EMA using mifepristone-misoprostol a real possibility for a sizeable number of Australian women having to make the decision about abortion for themselves. Nevertheless, there are some aspects of their reported outcomes which concern us, and which have major implications for the national provision of mifepristone, particularly as it appears that since our initial efforts, home abortion has become the preferred option for Australian providers.

When the practitioner providing the abortion is not the person who will provide emergency care for any complications (and we agree with the authors that complications are uncommon), it is mandatory that accessible and well documented prior arrangements are in place for such care; access to a telephone helpline alone is insufficient, especially for women in rural areas. Recent guidelines recommend this. ${ }^{3}$ While the clinical services required for the management of complications of EMA are exactly those required for spontaneous miscarriage, women who have undergone abortion elsewhere may be much more reluctant to access these services locally than are women presenting with spontaneous miscarriage. This will be particularly the case where a woman has travelled away from home to access abortion specifically because she does not wish her family and/or local health care provider to be aware of the abortion.

Not all women requesting EMA are suitable candidates for home abortion. Some women in poor socioeconomic circumstances and those who cannot find a suitable support person may be better served by surgical abortion. We should look too at the provision of EMA in day-surgery settings for women travelling long distances to access abortion, particularly for women with gestations of $>7$ weeks. A large majority of the women reported in the current study were $<7$ weeks. It is well documented that later gestations are associated with more bleeding and pain, and a higher rate of complications. ${ }^{3,9}$ The maximum gestation for safe home abortion is 9 weeks; at later gestations, medical abortion must take place in hospital. ${ }^{1-6}$
Either prophylactic antibiotics, or pre-abortion screening for sexually transmitted infections and treatment of detected cases, should be part of all protocols. We urgently need appropriate randomised controlled trials to determine the most effective approach, but the possibility of client non-compliance with antibiotic regimens also needs to be factored in. Every effort should be made to follow up women having an EMA at home, both for assurance of complete and uncomplicated outcomes, and to provide effective ongoing contraception.

Early surgical abortion is now very safe in Australia, with less than one death per 100000 women being the norm. ${ }^{8}$ Hopefully, a drug company may soon receive TGA approval to market mifepristone nationally. We look forward to EMA being available to all Australian women who request it, and wish to see EMA recognised as being as safe as the surgical alternative.

Competing interests: No relevant disclosures.

Provenance: Commissioned; externally peer reviewed.

1 Fiala C, Gemzel-Danielsson K. Review of medical abortion using mifepristone in combination with a prostaglandin analogue. Contraception 2006; 74 : 66-86.

2 Kulier R, Kapp N, Gulmezoglu AM, et al. Medical methods for first trimester abortion. Cochrane Database Syst Rev 2011; (11): CD002855. doi: 10.1002/ 14651858.CD002855.pub4.

3 Royal College of Obstetricians and Gynaecologists. The care of women requesting induced abortion. Evidence-based clinical guideline number 7. London: RCOG, 2011. http://www.rcog.org.uk/files/rcog-corp/ Abortion\%20guideline_web_l.pdf (accessed Aug 2012).

4 de Costa CM, Russell DB, de Costa NR, et al. Early medical abortion in Cairns, Queensland: July 2006 - April 2007. Med J Aust 2007; 187: 171-173.

5 World Health Organisation Task Force on Post-ovulatory Methods of Fertility Regulation. Comparison of two doses of mifepristone in combination with misoprostol for early medical abortion: a randomised trial. BJOG 2000; 107: 524-530.

6 Schaff EA, Fielding SL, WesthoffC. Randomised trial of oral versus vaginal misoprostol 2 days after mifepristone $200 \mathrm{mg}$ for abortion up to 63 days of pregnancy. Contraception 2002; 66: 247-250.

7 Fiala C, Winikoff B, Helstrom L, et al. Acceptability of home use of misoprostol in medical abortion. Contraception 2004; 70: 387-392.

8 Mulligan E, Messenger H. Mifepristone in South Australia - the first 1343 tablets. Aust Fam Physician 2011; 40: 342-345.

9 de Costa C. Medical abortion: the Australian experience. Expert Rev Obstet Gynecol 2012; 7: 25-30.

10 Goldstone P, Michelson J, Williamson E. Early medical abortion using low-dose mifepristone followed by buccal misoprostol: a large Australian observational study. Med J Aust 2012; 197: 282-286. 